\title{
Ants Are Not Conscious
}

\author{
Russell K. Standish \\ School of Mathematics and Statistics, The University of New South Wales, Sydney, Australia \\ Email: hpcoder@hpcoders.com.au \\ Received September 10 $0^{\text {th }}, 2012$; revised October 15 $5^{\text {th }}, 2012$; accepted October $25^{\text {th }}, 2012$
}

\begin{abstract}
Anthropic reasoning is a form of statistical reasoning based upon finding oneself a member of a particular reference class of conscious beings. By considering empirical distribution functions defined over animal life on Earth, we can deduce that the vast bulk of animal life is unlikely to be conscious.
\end{abstract}

Keywords: Anthropic Reasoning; Consciousness; Damuth's Law; Power Law

\section{Introduction}

Consciousness is a bête noir of the physical sciences. Each and every one of us is aware of his or her own consciousness, and indeed it seems to be necessary in order to carry out science, or at very least to give meaning to its theories and results. Yet, the more neurophysiologists probe the workings of the brain, the more of a phantom consciousness appears to be. Some argue that even if a complete neurophysical theory of the brain's function is determined, the "hard" problem of how phenomenal experience is generated still remains (Chalmers, 1995). Related to this issue is that we cannot prove definitively that any other individual of the human race is conscious and not a zombie that acts for all intents and purposes as conscious. Consciousness is fundamentally a first-person phenomenon with scientific discourse relegated to comparing reports with our own experience. Yet it is unreasonable to doubt the consciousness of other humans, who are constructed in the same way as ourselves, and who act in the same way as ourselves. The same is not true of other species, who are constructed from different body plans, have very different neural structures, and act in significantly different ways to ourselves. In the words of Nagel (1974) "What is it like to be a bat?" answering the question of consciousness in animals seems hopeless. Whilst Nagel was assuming that it is something to be like a bat, it is entirely reasonable to ask the question of whether it is anything to be like a bat. Most people would assume that on a scale of organism complexity from human beings, through vertebrates, invertebrates, etc. through to non-living matter, a line can be drawn between organisms experiencing phenomenal consciousness and those that don't. Descartes, for example, drew the line between humans and non-humans. Others would argue that some other species of mammal, and possibly bird as well as some cephalopods are probably conscious. Some even argue that insects might be conscious (Tye, 1997).

In this paper, I define consciousness in an operational way by noting that the reference class of anthropic reasoning (Bostrom, 2002) must consist of conscious entities, possibly restricted in some way, such as the set of terrestrial animals. Anthropic reasoning is best known in the form of the Cosmological Anthropic Principle (Barrow \& Tipler, 1986) and the infamous Doomsday Argument (Leslie, 1989).

Anthropic reasoning has been criticized on a number of fronts, particularly where it has been applied to produce counter intuitive conclusions. For example, the fine tuning argument has been used as evidence for a divine creator, or as evidence for a multi-verse, and the doomsday argument suggests that the human population will crash in the not too distant future. Most of these objections have been rebutted in Bostrom's book (Bostrom, 2002), who makes a well-argued case that anthropic reasoning can be done validly. It is not the purpose of this paper to review to the structure of these arguments, objections raised, nor rebuttals of those objections, as that is incidental to the aims of this paper. However, two issues in particular are pertinent: the reference class problem, and the measure problem.

The issue of what constitutes the class of observers from which the subject observer reasons he/she was randomly sampled is known as the reference class problem. For many examples of Anthropic Reasoning, precisely what constitutes the reference class does not bear much on the conclusions of the reasoning. Bostrom (2002) gives examples of this. In this paper, we very much turn the reasoning on it head, and ask what can we establish about the reference class, given the observation of what we are, and other information we might have at hand.

It might be argued that the reference class used for anthropic reasoning should only include those observers capable of understanding anthropic arguments, or more widely, those conscious entities capable of introspection. It is not at all clear whether this would include all humans, just a subset of humans, or non-human species as well. Conversely, the widest possible reference class is the set of all conscious observers, the interpretation I wish to use here. An alternative reading of this paper is that it is not talking about consciousness per se, but what is, or is not, allowable within the anthropic reference class.

The measure problem comes from extending anthropic reasoning to infinite sets of observers, such as we would expect to be the case in a multi-verse. In the set $\mathbb{N}=\{0,1,2, \cdots\}$, we might be tempted to say that the set of even numbers has measure 0.5 . Yet if we write the set in a different order as $\mathbb{N}=\{0,1,3,5,2,7,9,11,4, \cdots\}$, the same line of argument produces a measure of 0.25 . However, with respect to the arguments given in this paper, this measure problem doesn't arise, as the measure is already known empirically.

To consider the title question of this paper, we only need to note that there are considerably more ants than humans in the world. It is estimated that ants monopolize between $15 \%-20 \%$ of the terrestrial animal biomass (Schultz, 2000), far exceeding 
that of the vertebrates. A typical suburban house garden will contain city-scale populations of ants. A nave application of anthropic reasoning would conclude that ants could not be conscious, as otherwise we would expect to be an ant rather than a human.

Unfortunately this usage of anthropic reasoning raises the Chinese paradox. Why wasn't I born in the most populous nation on Earth, China, which has 50 times the population of my country of birth, Australia?

Furthermore, ants are not a single species, but are taxonomically speaking a family, with which we are comparing a single species homo sapiens. It would be better to rephrase the question in a way that didn't depend on a somewhat human-biased taxonomic scheme.

In the rest of this paper I show that the Chinese paradox is actually not a problem for anthropic reasoning, and in so doing demonstrate a previously unknown process for generating power law distributions. Then by recasting the ant consciousness problem into a question of expected body mass, which is an objective physical measurement rather than a possibly subjective classification, and including the proper handling of the Chinese paradox, we can conclude that the vast majority of animal species (particularly the small ones) are unlikely to be conscious (or in the reference class, if you prefer) by anthropic reasoning.

\section{Chinese Paradox}

China has well over a billion people, and along with India, has by far the biggest population of all the nations in the world. I happen to live in Australia, for instance, a country with around $1 / 50$ th the population of China. It would be absurd to conclude that Chinese people are unconscious, so namely one would expect on anthropic grounds to be Chinese or Indian. At first sight this looks disastrous for anthropic reasoning, until you realize that it is ill-posed. Suppose you asked the question of what is the chance of being Chinese versus not being Chinese. There is about a $20 \%$ chance of being Chinese, and $80 \%$ not, so it then becomes unsurprising to not be Chinese.

We can rephrase the Chinese question in a different way: What is the expected population size of one's country of birth? It turns out (see Figure 1) that there are far more countries with fewer people, than countries with more people. The relationship between population size and the number of countries looks roughly proportional to $1 / x$, where $x$ is the population of the country. This law is an example of a power law, and it appears in all sorts of circumstances, for example the frequency with which words are used in the English language.

With a $1 / x$ power law, the number of countries of a given population size exactly offsets the population of those countries, so anthropically speaking, we should expect to find ourselves in just about any sized country, with the same probability. Being in a country with a population the size of Australia's would be no more surprising than being in a more populated country such as the US or China.

However, the actual distribution of country populations turns out to be a $\log$ normal distribution ${ }^{1}$, whose probability distribution is

$$
p(x)=C / x \exp \left(-(\ln x-\mu)^{2} / 2 \sigma^{2}\right)
$$

\footnotetext{
${ }^{1}$ Thank you to Aaron Clauset for pointing this out.
}

The parameters $\mu$ and $\sigma$ can be found by means of the maximum likelihood method outlined by Clauset et al. (2009). In fact one can compare the likelihood of the lognormal distribution explaining the population data with the likelihood that the $1 / x$ power law explains it, and it turns out to be of the order of $10^{11}$ times as likely. Similar results hold for other population datasets in the range 1965-2005. So indeed it would be more likely for one to find oneself in a middle ranked country like Kuwait or Estonia, than in the most populous nations of India or China. However, the effect is not marked. India and China together have about 2.4 billion people, and the total number of people living in countries with populations in the range 10 100 million is about 2.1 billion, and in the range 100 million to a billion is about 1.5 billion.

Given the ubiquity of power laws, and the fact that a $1 / x$ power law exactly neuters any observer selection effect as in the above case, might a $1 / x$ power law be a signature of an arbitrary, or random classification?

\section{Mass Distribution of Animal Species}

OK, well let's get back to our ants, and ask the question of what is the expected abundance of our species, assuming we are randomly sampled from all conscious species on the Earth. The distribution of species populations tends to follow a power law, with a typical rank-abundance plot within a species size class following a power law $A \propto r^{m}$ with exponent $m=-1.9$ (Siemann et al. 1999), where $A$ is the abundance of the the $r$ th most abundant species. Rank-abundance plots are related to cumulative size distributions (Newman, 2005):

$$
r(A)=N \int_{A}^{\infty} p(x) \mathrm{d} x
$$

where $N$ is the total number of species in a size class, and $p(x)$ the distribution of species abundances. Solving (2) implies $p(A)$ is also a power law, with exponent -1.52 . By the argument in the previous section, we would therefore expect to find ourselves to be one of the many species with few individuals, if all animals were conscious, as the distribution of abundances falls off faster than $1 / A$. Yet our species abundance is many orders higher $\left(6 \times 10^{9}\right)$ than the minimum abundance for viability (approx $10^{3}$ ). However, for the most part of our species' existence on the Earth, our abundance was much less, and perhaps integrated over time, our total abundance is not so different from that of other species of our size class.

However, let us ask a different question: "what is our expected body mass if we are randomly sampled from the reference class of conscious beings?" For this we need the abundance distribution $P(m)$ as a function of body mass.

There is a well known biological law (called Damuth's law) (Damuth, 1991) that states the population density of a species is inversely proportional to the $3 / 4$ ths power of that species' body mass, i.e. $A \propto m^{-3 / 4}$. To turn this result into the mass distribution of individuals $P(m)$, we need to multiply this law by the mass distribution of species $S(m)$. Informally, we note that there are many more smaller bodied species of animals than larger ones; there are many more types of insect than of mammals, for example. The exact form of the distribution function $S(m)$ is still a matter of conjecture. Some theoretical models suggest that $S(m)$ is peaked at intermediate body sizes (Hutchinson \& MacArthur, 1959), and experimental results appear to confirm this (Siemann et al. 1999), although it must be admitted that the latter study was confined to insects, and ignored 


\section{R. K. STANDISH}

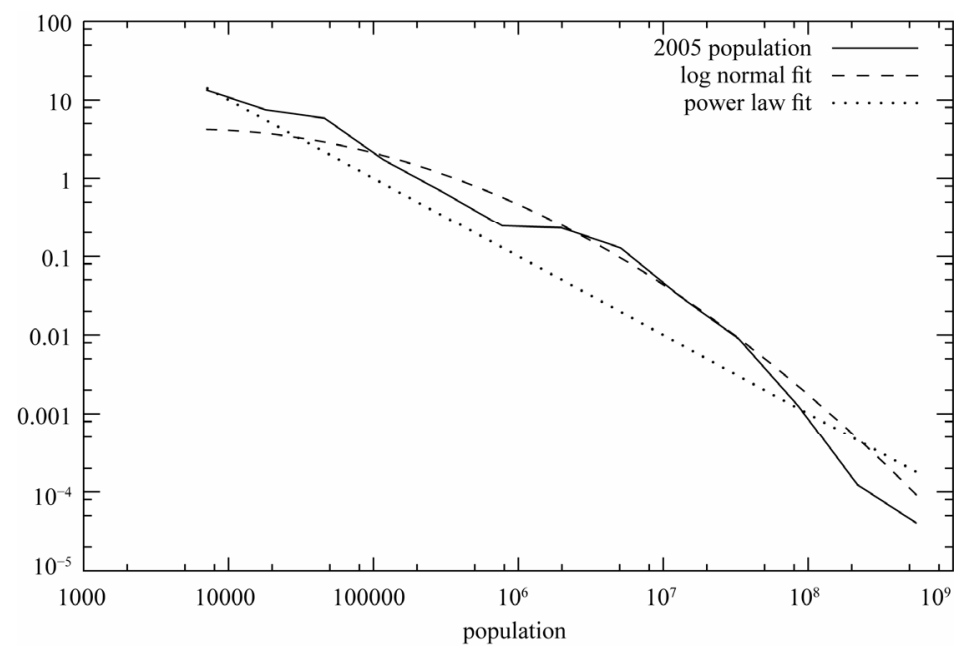

Figure 1.

Distribution of national populations in the year 2005, plotted on a log-log scale (US Census Bureau, 2005). Also plotted are the best fits for a power law (slope -1.05) and lognormal $(\mu=14.8, \sigma=2.5)$.

the huge diversity of nematodes. Of more interest was the finding that $S(m) \propto P(m)^{0.5}$ Siemann et al. 1999 (Siemann et al. use $I(m)$ instead of $P(m))$. Writing

$$
P(m) \propto S(m) m^{-3 / 4} \propto P(m)^{1 / 2} m^{-3 / 4}
$$

we can solve for $P(m)$ as

$$
P(m) \propto m^{-3 / 2}
$$

By the same arguments as above, we should expect to find ourselves near the lower body mass of the class of conscious animals, ruling out the vast majority of animals that are insects etc.

\section{Bayesian Formulation}

The argument can be cast in a Bayesian framework in the following way. Let $A$ represent the hypothesis that all animals are conscious, and $B$ represent the observation that our observed body mass is greater than (for arguments sake) $10 \mathrm{~kg}$.

The previous argument could be criticised as suffering from what is known as the "Prosecutor's fallacy". The value $p(B \mid A)$ can be computed from (4) by integration:

$$
\begin{aligned}
p(B \mid A) & =\int_{10 \mathrm{~kg}}^{\infty} P(m) \mathrm{d} m \\
& =\left(\frac{10 \mathrm{~kg}}{m_{0}}\right)^{-1 / 2} \\
& \approx 10^{-5} \text { with } m_{0}=1 \mu \mathrm{g}
\end{aligned}
$$

where $m_{0}$ is the minimum mass of a conscious animal under hypothesis $A$. A suitable choice for such an animal is $C$. elegans, a $1 \mathrm{~mm}$ long nematode with a nervous system consisting of 302 neurons. The mass of an adult C. elegans is around $2 \mu \mathrm{g}$ (Knight et al., 2002). Even if we were to limit the discussion to animals of the size of ants (our titular species) or bigger (60 $\mu \mathrm{g}$ $2 \mathrm{mg}$ (Kaspari, 2005)), $p(B \mid A) \approx 10^{-4}$.

However, the question we really want to know the answer to is what is $p(A \mid B)$ - what is the likelihood of all animals being conscious, given that our observed body mass is more than 10 $\mathrm{kg}$ ?

Bayes law is written as

$$
p(A \mid B)=\frac{p(B \mid A) p(A)}{p(B)}
$$

The term $p(A)$ represents our prior conviction in $A$. We can, for the sake of argument, assume $p(A)=1$ here. Any lesser value only increases the force of this argument.

The final term $p(B)$ is the probability of observing one's body mass greater than $10 \mathrm{~kg}$. Since we don't know the mass distribution of conscious beings, we cannot calculate this value directly. However, we can use a form of anthropic reasoning introduced by Gott III (1994). In that case, Gott argued that a number drawn at random from a uniform distribution on the numbers $1 \cdots N$ would find its value to lie in the range $(0.5 N, N]$ with confidence $95 \%$. Suppose instead that the numbers were ordered according to some attribute $m$, drawn from some unknown distribution $M(m)$. Then by the same argument, we can say that with $95 \%$ confidence

$$
\int_{m}^{\infty} M\left(m^{\prime}\right) \mathrm{d} m^{\prime}>0.05 .
$$

But the left hand side of (9) with $m=10 \mathrm{~kg}$ is just our term $p(B)$, where $M(m)$ is the unknown distribution of masses of conscious observers. Thus with confidence $c \in[0,1)$, we can assume $p(B)>1-c$.

Plugging this into (8), our confidence in hypothesis $A$ being wrong is

$$
c=1-\sqrt{p(B \mid A)}
$$

which is $99.7 \%$ for nematodes and $99 \%$ for ants. By contrast, for the proposition that all mammals are conscious, our confidence in this being wrong by (10) is only about $90 \%$, using the smallest known mammal mass of about $2 \mathrm{~g}$ for the Pygmy Shrew. $90 \%$ is generally considered not statistically significant, so anthropic reasoning cannot be used to rule out the consciousness of all mammals without further refinement of $p(A)$. 


\section{R. K. STANDISH}

\section{Conclusion}

In this paper, the reference class of anthropic reasoning is used as a way to reason about the species of animals that could be conscious. Considering the reference class to be all conscious animals on the Earth, one applies known distributions of species abundances to determine that: one's nationality is not expected to be any particular country, owing to a $1 / x$ distribution of population sizes; that one's body mass should be near the lower limit of the set of conscious animals; and the abundance of one's own species should be near the lower limit of species abundances.

Considering our body mass is substantially higher than the average animal (who is an insect, or even possibly a nematode), we can conclude that the vast bulk of the animal kingdom is unlikely to be conscious. We might also conclude, based on the high present abundance of humans, that most species of our mass class are also not conscious, since we should also expect to find ourselves near the lower limit of species abundance of conscious species. But this would be a mistake-it is only natural, assuming we're born human, to be born in an era of high human abundance. Integrated over our entire species lifetime, the total human abundance may not be so different from that of other species in our size class.

\section{REFERENCES}

Barrow, J. D., \& Tipler, F. J. (1986). The anthropic cosmological principle. Oxford: Clarendon.

Bostrom, N. (2002). Anthropic bias: Observation selection effects in science and philosophy. New York: Routledge.

Chalmers, D. J. (1995). Facing up to the problem of consciousness.
Journal of Consciousness Studies, 2, 200-219.

Clauset, A., Shalizi, C. R., \& Newman, M. E. J. (2009). Power-law distributions in empirical data. SIAM Review, 51, 661-703. doi:10.1137/070710111

Damuth, J. (1991). Of size and abundance. Nature, 351, 268-269. doi: $10.1038 / 351268 \mathrm{a} 0$

Gott III, J. R. (1994). Future prospects discussed. Nature, 368, 108. doi: $10.1038 / 368108 \mathrm{a} 0$

Hutchinson, G. E., \& MacArthur, R. J. (1959). A theoretical ecological model of size distributions among species of animals. American Naturalist, 93, 117-125. doi:10.1086/282063

Kaspari, M. (2005). Global energy gradients and size in colonial organisms: Worker mass and worker number in ant colonies. PNAS, 102, 5079-5083. doi:10.1073/pnas.0407827102

Knight, C. G., Patel, M. N., Azevaedo, R. B. R., \& Leroi, A. M. (2002). A novel mode of ecdysozoan growth in C. elegans. Evolution and Development, 4, 16-27. doi:10.1046/j.1525-142x.2002.01058.x

Leslie, J. (1989). Universes. New York: Routledge.

Nagel, T. (1974). What is it like to be a bat? The Philosophical Review, 83, 435-450. doi:10.2307/2183914

Newman, M. E. J. (2005). Power laws, pareto distributions and zipf's law. Contemporary Physics, 46, 323-351. doi:10.1080/00107510500052444

Schultz, T. R. (2000). In search of ant ancestors. PNAS, 97, 1402814029. doi: $10.1073 /$ pnas. 011513798

Siemann, E., Tilman, D., \& Haarstad, J. (1999). Abundance, diversity and body size: Patterns from a grassland arthropod community. Journal of Animal Ecology, 68, 824-835. doi:10.1046/j.1365-2656.1999.00326.x

Tye, M. (1997). The problem of simple minds: Is there anything it is like to be a honey bee? Philosophical Studies, 88, 289-317. doi:10.1023/A:1004267709793

US Census Bureau (2005). IDB—Rank countries by population. http://www.census.gov/ipc/www/idbrank.html 